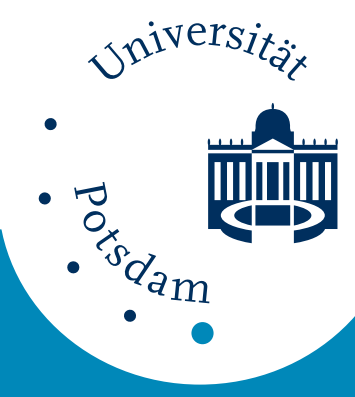

Universität Potsdam

Peter Maaß, Sergei V. Pereverzev, Ronny Ramlau, Sergei G. Solodky

An adaptive discretization for TikhonovPhillips regularization with a posteriori parameter selection

NLD Preprints ; 48 


\title{
An adaptive discretization for Tikhonov-Phillips regularization with a posteriori parameter selection
}

\author{
Peter Maaß* Sergei V.Pereverzev Ronny Ramlau \\ Sergei G.Solodky
}

\begin{abstract}
The aim of this paper is to describe an efficient adaptive strategy for discretizing ill-posed linear operator equations of the first kind: we consider Tikhonov-Phillips regularization

$$
x_{\alpha}^{\delta}=\left(A^{*} A+\alpha I\right)^{-1} A^{*} y^{\delta}
$$

with a finite dimensional approximation $A_{n}$ instead of $A$. We propose a sparse matrix structure which still leads to optimal convergences rates but requires substantially less scalar products for computing $A_{n}$ compared with standard methods.
\end{abstract}

\section{Introduction}

The aim of this paper is to describe an adaptive strategy for discretizing ill-posed linear operator equations of the first kind

$$
A x=y .
$$

We assume that only perturbed data $y^{\delta}$ with $\left\|y-y^{\delta}\right\|$ is available. More precisely, we consider Tikhonov-Phillips regularization

$$
x_{\alpha}^{\delta}=\left(A^{*} A+\alpha I\right)^{-1} A^{*} y^{\delta}
$$

*This work has been partially supported by the DFG under grant MA 1657/1-2 
where the regularization parameter $\alpha$ is chosen according to Morozov's discrepancy principle, i.e. one determines the largest $\alpha \in\left\{\alpha_{m}=q^{m} \alpha_{0} \mid m=\right.$ $0,1, .$.$\} s.t.$

$$
\left\|A x_{\alpha}^{\delta}-y^{\delta}\right\| \leq d \delta
$$

Any numerical realisation requires to carry out all computations with a finite dimensional approximation $A_{n}$ instead of $A$.

The choice of the approximation $A_{n}$ determines the accuracy as well as the overall complexitiy of the algorithm. The complexity of the algorithm has to be measured in two categories: 1 ) the number of scalar products required to compute $\left.A_{n}, 2\right)$ the number of matrix-vector-multiplications-weighted by the number of non-zero entries of $A_{n}$ - required to compute (2).

Several authors have investigated approximations of the type

$$
A_{n}=Q A P \text {. }
$$

where $P$ and $Q$ denote orthogonal projections onto suitable finite dimensional subspaces. These investigations aim at minimizing the dimensions of the subspaces. E.g. [16] treats discretizations of this type for general projection methods and [12] investigates discretizations for Tikhonov regularization. A recent publication [7] also exploits the strutcure of the resulting linear systems for different values of $\alpha$ in order to construct efficient CG-methods for solving (2). However, all these publications link the level of approximation to the data error $\delta$ only, i.e. $A_{n}$ is kept fixed for all $\alpha$ which have to be tested. A first adaptive strategy, which linked the level of approximation to the value of $\alpha_{m}$ by

$$
\left\|A_{n}-A\right\| \leq c \sqrt{\alpha} \sqrt{\delta},
$$

was suggested in [10]. There the approximations were obtained by wavelet techniques in order to obtain sparse approximations. This allows for an efficient computation of the matrix-vector multiplications needed for deter$\operatorname{mining} x_{\alpha_{m}}^{\delta}$.

From a numerical point of view an efficient algorithm for computing the approximation $A_{n}$ is equally important. This efficiency (or complexity of type 1 ) is measured by the number of scalar products required to compute $A_{n}$. This number is called the required amount of discrete information.

If $A_{n}$ is obtained from a $2^{n}$-dimensional approximation space

$$
V_{n}=\operatorname{span}\left\{\varphi_{j} \mid j=1,2, . ., 2^{n}\right\}
$$




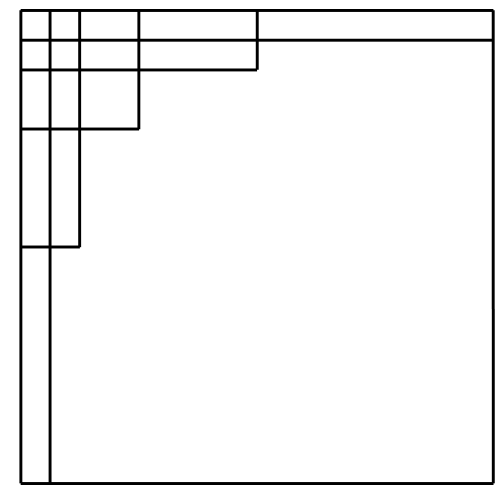

Figure 1: The marked area determines the coefficients of $A_{n}$ for $n=2$ which have to be computed in the adaptive discretization scheme. The dimnension of this matrix is $2^{2 n}=16$.

then standard methods require the evaluation of $2^{2 n}$ scalar products $\left\langle\varphi_{i}, A \varphi_{j}\right\rangle$ for computing $A_{n}$. This is the case even for wavelet-approximations if no further assumptions on the structure of $A$, e.g. convolution operator or weakly singular integral operators with known degree of singularity $[2,3]$, can be exploited.

The computation of such scalar products would be cheap if a singular value or a wavelet-vaguelette decomposition of $A[5,4]$ would be available. However the computation of these decompositions is in general as costly as solving $2^{n}$ linear systems of dimension $2^{n}$.

An elegant and efficient approximation was proposed in [14]. The number $n$ has to be even in this approach, i.e. the dimensional index is replaced by $2 n$ :

$$
A_{d i s c}=A_{n}=\sum_{j=1}^{2 n}\left(P_{j}-P_{j-1}\right) A P_{2 n-j}+P_{0} A P_{2 n},
$$

where $P_{j}$ denotes the orthogonal projection onto a suitable $2^{j}$ - dimensional subspace. This idea amounts to computing only a small fraction of the coefficients of the matrix $A_{n}$.

The idea of this paper is to combine the approaches in [14] and [10], ie. to combine the discretization (3) with an adative strategy for choosing $n$ depending on $\delta$ and $\alpha$. This strategy still yields optimal convergence rates but requires substantially less scalar products for computing the discretized operators, i.e. these methods require a smaller amount of discrete information. 


\section{Notation and basic assumptions}

Denote by $(\cdot, \cdot)$ the inner product for some Hilbert space $H$ and as usual, $\|\varphi\|_{H}=(\varphi, \varphi)^{1 / 2}$. For $r \in(0, \infty)$, we let $H^{r}$ denote a linear subspace of $H$ which is equipped with a norm $\|\varphi\|_{H^{r}} \geq\|\varphi\|_{H}$. Moreover, in the spirit of wavelets updating [3] we suppose that there exists a sequence of nested finite dimensional subspaces $V_{0} \subset V_{1} \subset V_{2} \subset \ldots \subset V_{\ell} \subset \ldots \subset H$ such that

$$
\inf _{v_{j} \in V_{j}}\left\|v-v_{j}\right\|_{H} \leq c_{r} 2^{-r j}\|v\|_{H^{r}}, \quad v \in H^{r}
$$

and

$$
\operatorname{dim} V_{j} \sim 2^{s j},
$$

where $s \geq 1$ and $a \sim b$ will always mean that $a$ and $b$ can be bounded by constant multiples of each other. The condition (4) can be written in the form

$$
\left\|I-P_{j}\right\|_{H^{r} \rightarrow H} \leq c_{r} 2^{-r j},
$$

with a constant $c_{r} \geq 1$ and where $P_{j}: H \rightarrow V_{j}$ is the orthogonal projection onto subspace $V_{j}$, i.e.

$$
P_{j} v=\sum_{k=1}^{\operatorname{dim} V_{j}}\left(v, \varphi_{j, k}\right) \varphi_{j, k} .
$$

Here $\Phi_{j}=\left\{\varphi_{j, k}: k=1,2, \ldots, \operatorname{dim} V_{j}\right\}$ denotes an orthonormal basis of $V_{j}$, $\Phi_{j-1} \subset \Phi_{j}$.

Now we define the class of equations (1) which will be considered in the sequel. First of all it will be assumed that the operators $A$ have some smoothness with respect to the family of subspaces $H^{r}, r \in(0, \infty)$. Namely,

$$
A \in \mathcal{H}_{\gamma}^{r}=\left\{A:\|A\|_{H \rightarrow H^{r}}+\left\|A^{*}\right\|_{H \rightarrow H^{r}} \leq \gamma\right\}, \quad \gamma \geq 1 .
$$

Here $A^{*}$ denotes the adjoint operator of $A: H \rightarrow H$, i.e. $(f, A g)=\left(A^{*} f, g\right)$ for any $f, g \in H$.

Now we present a rather simple example to illustrate the assumptions described above. We consider an integral equation

$$
A x(t):=\int_{0}^{1} k(t, \tau) x(\tau) d \tau=y(t) .
$$

The underlying spaces and projections are chosen as follows. As Hilbert space $H$ we take the space $L_{2}(0,1)$ with the usual norm and inner product. 
As $H^{r}$ we take the Sobolev space $W_{2}^{r}(0,1)$ of functions $f(t)$ having squaresummable derivatives $f^{(i)} \in L_{2}(0,1), i=0,1, \ldots, r$, with an appropriate norm. For $r=1$ as nested finite dimensional subspaces we choose the spaces of piecewise constant functions, such that

$$
\begin{gathered}
V_{0}=\operatorname{span}\{1\}, \quad V_{j}=\operatorname{span}\left\{\varphi_{j, k}, \quad k=1,2, \ldots, 2^{j}\right\}, \\
\operatorname{dim} V_{j}=2^{j} .
\end{gathered}
$$

Here $\left\{\varphi_{j, k}\right\}$ is the orthonormal basis of Haar-wavelet functions, where $\varphi_{j, 1}=1$ and for $k=2^{m-1}+i, m=1,2, \ldots, j, i=1,2, \ldots, 2^{m-1}$

$$
\varphi_{j, k}(t)= \begin{cases}2^{(m-1) / 2}, & t \in\left[(i-1) / 2^{m-1},(i-1 / 2) / 2^{m-1}\right) \\ -2^{(m-1) / 2}, & t \in\left[(i-1 / 2) / 2^{m-1}, i / 2^{m-1}\right) \\ 0, & \text { elsewhere }\end{cases}
$$

It is well-known that for such spaces $V_{j}$ and for the orthonormal projection $P_{j}: L_{2}(0,1) \rightarrow V_{j}$ we have

$$
\left\|I-P_{j}\right\|_{W_{2}^{1}(0,1) \rightarrow L_{2}(0,1)} \leq c_{1} 2^{-j} .
$$

This means that for $H=L_{2}(0,1), H^{r}=W_{2}^{r}(0,1)$ and for spaces of Haarwavelet functions $V_{j}$ the conditions (4)-(6) hold for $r=1, s=1$. As indicated in [3], for example, one can construct wavelets on the interval $[0,1]$ in such a manner that (5), (6) hold for $H=L_{2}(0,1), H^{r}=W_{2}^{r}(0,1), r>1, s \geq 1$. If the kernel $k(t, \tau)$ of the integral operator of (8) has square-summable partial derivatives $\frac{\partial^{i} k(t, \tau)}{\partial t^{i}}, \frac{\partial^{i} k(t, \tau)}{\partial \tau^{i}}, i=0,1, \ldots, r$, then it is easy to see that $A \in \mathcal{H}_{\gamma}^{r}$ for $H=L_{2}(0,1), H^{r}=W_{2}^{r}(0,1)$ and some $\gamma$.

We shall study the equation (1) with $A \in \mathcal{H}_{\gamma}^{r}$. On the other hand, from the condition (7) one sees that $A \in \mathcal{H}_{\gamma}^{r}$ is a compact linear operator acting from $H$ to $H$ and so it is not continuously invertible. In this setting the problem (1) is ill-posed, that is, its minimum norm solution $x^{\dagger}$ does not depend continuously on the right-hand side $y$. Small perturbation $y_{\delta}$ of the exact but unknown data $y$ may cause dramatic changes in $x^{\dagger}$.

The usual discussion of the order of accuracy of solution techniques for (1) is done under the assumptions that the minimum norm solution $x^{\dagger}$ lies 
in the range of $\left(A^{*} A\right)^{\nu}, \nu>0$, that is

$$
x^{\dagger}=\left(A^{*} A\right)^{\nu} v, \quad\|v\|_{H} \leq \rho,
$$

and the perturbed data $y_{\delta}$ satisfies $\left\|y-y_{\delta}\right\|_{H} \leq \delta$ with an a priori known noise level $\delta>0$. From [17], [18] it follows that under these assumptions for any solution technique the best possible order of accuracy in the power scale is $\delta^{2 \nu /(2 \nu+1)}$. Therefore in the sequel we shall consider the class $\Phi_{\gamma, \rho}^{r, \nu}$ of equations (1) with $A \in \mathcal{H}_{\gamma}^{r}$ and

$$
y \in A M_{\nu, \rho}(A)=\left\{f: \quad f=A u, \quad u \in M_{\nu, \rho}(A)\right\},
$$

where $M_{\nu, \rho}(A)=\left\{u: u=\left(A^{*} A\right)^{\nu} v,\|v\|_{H} \leq \rho\right\}$. It is easy to see that in this case the solution $x^{\dagger}$ of (1) has the form (9).

\section{Morozov's discrepancy principle for the stan- dard projection methods with a predeter- mined level of discretization}

Traditionally the discretization of problem (1) is done by a Galerkin method. This means that instead of (1) we consider now the equation

$$
A_{d i s c} x=P_{m} y_{\delta}
$$

where $A_{d i s c}=P_{m} A P_{n}$. But if (1) is ill-posed, i.e., the solution $x$ is not a continuous function of the data $y$, regularization techniques are required for solving (10). In this paper we consider Tikhonov-Phillips regularization. In this method a solution of (10), and hence of (1), is approximated by a solution $x_{\alpha, m, n}$ of the equation

$$
\alpha x+A_{d i s c}^{*} A_{d i s c} x=A_{d i s c}^{*} y_{\delta} .
$$

Note that finding on element $x_{\alpha, m, n}$ reduces to solving a system of $\min \left\{\operatorname{dim} V_{m}\right.$, $\left.\operatorname{dim} V_{n}\right\}$ linear algebraic equations.

One of the most widely used strategies for choosing the regularization parameter $\alpha$ is Morozov's discrepancy principle [11]. Following [16], we shall consider this discrepancy principle in a form tailored to the discretized version of Tikhonov-Phillips regularization and $A \in \mathcal{H}_{\gamma}^{r}$ : Let $1<d_{1} \leq d_{2}$. If 
$\left\|P_{m} y_{\delta}\right\|_{H} \leq d_{1} \delta$, then take $x=0$ as the approximation. If $\left\|P_{m} y_{\delta}\right\|_{H}>d_{1} \delta$, then choose $\alpha \geq \alpha_{\min }=\left(\gamma c_{r} 2^{-n r}\right)^{2}$ such that

$$
d_{1} \delta \leq\left\|P_{m} y_{\delta}-A_{d i s c} x_{\alpha, m, n}\right\|_{H} \leq d_{2} \delta .
$$

If there is no $\alpha \geq \alpha_{\min }$ such that (12) holds, then choose $\alpha=\alpha_{\min }$. The following theorem allows us to estimate the efficiency of the traditional approach to discretization (10)-(12).

Theorem 1 [16]. Let the parameter $\alpha$ be chosen according to the discrepancy principle (12). If equation (1) belongs to the class $\Phi_{\gamma, \rho}^{r, \nu}, 0<\nu \leq 1 / 2$, then

$$
\left\|x^{\dagger}-x_{\alpha, m, n}\right\|_{H} \leq d_{\nu}\left\{\delta^{\frac{2 \nu}{2 \nu+1}}+2^{-2 \nu n r}+2^{-2 \nu m r}\right\},
$$

where $d_{\nu}$ is independent of $\delta, n, m$.

Let us consider the following situation. We know that equation (1) belongs to the class $\Phi_{\gamma, \rho}^{r, \nu}$ for some $\nu \in(0,1 / 2]$, but we don't know the exact value of $\nu$. From Theorem 1 it follows that - within the framework of the standard projection methods (10)-(12) and with a predetermined level of discretization - we can guarantee the optimal order of accuracy $\delta^{2 \nu /(2 \nu+1)}$ in the case when $2^{n} \geq \delta^{-1 /(2 \nu+1) r}, 2^{m} \geq \delta^{-1 /(2 \nu+1) r}$ for all $\nu \in(0,1 / 2]$. As with [16], the minimal $m$ and $n$ satisfying above conditions for all $\nu \in(0,1 / 2]$ have the following orders: $2^{n} \sim 2^{m} \sim \delta^{-1 / r}$.

Denote by $\operatorname{card}(I P)$ the number of inner products of the form

$$
\left(\varphi_{m, k}, A \varphi_{n, k}\right) \quad \text { and } \quad\left(\varphi_{m, k}, y_{\delta}\right)
$$

required to construct an approximate solution $x_{\alpha, m, n}$ realizing the optimal order of accuracy for all $\nu \in(0,1 / 2]$. Then by virtue of $(5)$

$$
\operatorname{card}(I P)=\operatorname{dim} V_{m}\left(\operatorname{dim} V_{n}+1\right) \sim 2^{s(m+n)} \sim \delta^{-2 s / r} .
$$

\section{An adaptive discretization scheme}

So far we have discussed to which extend $A$ may be replaced by a discretized operator $A_{d i s c}$, where $A_{d i s c}$ is kept fixed for all possible values of the regularization parameter $\alpha$. However since we choose $\alpha$ by testing different values 
of the regularization parameter we would like to link the amount of discrete information $\operatorname{card}(I P)$ to $\alpha$. This will allow us to use coarser discretizations for large values of $\alpha$ and to obtain the optimal order of accuracy in the power scale $\delta^{2 \nu /(2 \nu+1)}$ using an amount of discrete information of the form (13) such that

$$
\operatorname{card}(I P) \sim(\delta \sqrt{\alpha})^{-s / r} \log ^{1+s / r}(\delta \sqrt{\alpha})^{-1} .
$$

Let us consider the discretization scheme within the framework of which

$$
\begin{aligned}
A_{d i s c} & =A_{n}=\sum_{j=1}^{2 n}\left(P_{j}-P_{j-1}\right) A P_{2 n-j}+P_{0} A P_{2 n}= \\
& =\sum_{j=1}^{2 n} P_{2 n-j} A\left(P_{j}-P_{j-1}\right)+P_{2 n} A P_{0} .
\end{aligned}
$$

Note that this scheme was used earlier in [15], p.295, for discretizing second kind operator equations (well-posed problems). This discretization uses a discretization space of dimension $2^{2 n}$ but computes only a small fraction of the scalar products required to compute the standard discretization $P_{2 n} A P_{2 n}$. To be more precise, this approximation incorporates the full discretization $P_{m} A P_{m}$ only for $m=n$ and adds some coefficients describing the mixing of high and low frequency components by the action of $A$.

In the sequel we need the lemma

Lemma 1 For $A \in \mathcal{H}_{\gamma}^{r}$

$$
\begin{gathered}
\left\|A^{*} A-A_{n}^{*} A_{n}\right\|_{H \rightarrow H} \leq c_{r, \gamma} n 2^{-2 n r}, \quad\left\|A A^{*}-A_{n} A_{n}^{*}\right\|_{H \rightarrow H} \leq c_{r, \gamma} n 2^{-2 n r}, \\
\left\|\left(A^{*}-A_{n}^{*}\right) A\right\|_{H \rightarrow H} \leq c_{r, \gamma} n 2^{-2 n r}
\end{gathered}
$$

where $c_{r, \gamma}=2^{r+3} c_{r}^{2} \gamma^{2}$.

Proof. We prove only the first estimate. Other estimates are established in a similar manner.

It is easy to see that

$$
\begin{gathered}
\left\|A^{*} A-A_{n}^{*} A_{n}\right\|_{H \rightarrow H} \leq\left\|A^{*}\left(I-P_{2 n}\right)^{2} A\right\|_{H \rightarrow H}+ \\
+\left\|A^{*} P_{2 n} A-A_{n}^{*} A_{n}\right\|_{H \rightarrow H} .
\end{gathered}
$$


Keeping in mind that

$$
A_{n}^{*} A_{n}=\sum_{j=1}^{2 n} P_{2 n-j} A^{*}\left(P_{j}-P_{j-1}\right) A P_{2 n-j}+P_{2 n} A^{*} P_{0} A P_{2 n},
$$

we have

$$
\begin{aligned}
\left\|A^{*} P_{2 n} A-A_{n}^{*} A_{n}\right\|_{H \rightarrow H} & \leq\left\|A^{*} P_{0} A-P_{2 n} A^{*} P_{0} A P_{2 n}\right\|_{H \rightarrow H}+ \\
& +\sum_{j=1}^{2 n}\left\|G_{j}\right\|_{H \rightarrow H},
\end{aligned}
$$

where

$$
G_{j}=A^{*}\left(P_{j}-P_{j-1}\right) A-P_{2 n-j} A^{*}\left(P_{j}-P_{j-1}\right) A P_{2 n-j} .
$$

It is easy to calculate that

$$
\begin{gathered}
\left\|G_{j}\right\|_{H \rightarrow H} \leq\left\|\left(I-P_{2 n-j}\right) A^{*}\left(P_{j}-P_{j-1}\right) A\right\|_{H \rightarrow H}+ \\
+\left\|P_{2 n-j} A^{*}\left(P_{j}-P_{j-1}\right) A\left(I-P_{2 n-j}\right)\right\|_{H \rightarrow H} \leq \\
\leq 2\left\|I-P_{2 n-j}\right\|_{H^{r} \rightarrow H}\left\|A^{*}\right\|_{H \rightarrow H^{r}}\left\|P_{j}-P_{j-1}\right\|_{H^{r} \rightarrow H}\|A\|_{H \rightarrow H^{r}} \leq \\
\leq 2 c_{r}^{2} \gamma^{2} 2^{-(2 n-j) r}\left(2^{-j r}+2^{-(j-1) r}\right)=2 c_{r}^{2} \gamma^{2}\left(1+2^{r}\right) 2^{-2 n r}, \\
\left\|A^{*} P_{0} A-P_{2 n} A^{*} P_{0} A P_{2 n}\right\|_{H \rightarrow H} \leq 2 c_{r} \gamma^{2} 2^{-2 n r}, \\
\left\|A^{*}\left(I-P_{2 n}\right)^{2} A\right\|_{H \rightarrow H} \leq c_{r}^{2} \gamma^{2} 2^{-4 n r} .
\end{gathered}
$$

Then by virtue of (16), (18) we obtain the assertion of the lemma.

Let us study the approximation properties of Tikhonov-Phillips algorithm with a parameter selection according to discrepancy principle which has the following form:

1. given data: $A \in \mathcal{H}_{\gamma}^{r}, y_{\delta}, \delta$;

2. initialization: $\alpha_{0}, 0<q<1, d>2$;

3. iterate 
(a) $\alpha=\alpha_{m}=q^{m} \alpha_{0}$,

(b) determine a discretization level $n=n(\alpha, \delta)$ such that

$$
n 2^{-2 n r}=4 c_{r, \gamma, \rho}^{-1} \delta \sqrt{\alpha_{m}},
$$

(c) compute the inner products

$$
\left(\varphi_{2 n, i}, y_{\delta}\right), \quad i=1,2, \ldots, \operatorname{dim} V_{2 n}
$$

(d) compute the inner products

$$
\begin{aligned}
&\left(\varphi_{j, k}, A \varphi_{2 n-j, \ell}\right), \quad j=1,2, \ldots, 2 n \\
& k=1,2, \ldots, \operatorname{dim} V_{j}, \\
& \ell=1,2, \ldots, \operatorname{dim} V_{2 n-j}
\end{aligned}
$$

required to construct $A_{n}$,

(e) compute $x_{\alpha_{m}, n}^{\delta}=\left(\alpha_{m} I+A_{n}^{*} A_{n}\right)^{-1} A_{n}^{*} y_{\delta}$ by solving

$$
\alpha_{m} x+A_{n}^{*} A_{n} x=A_{n}^{*} y_{\delta}
$$

until

$$
\left\|A_{n} x_{\alpha_{m}, n}^{\delta}-y_{\delta}\right\|_{H} \leq d \delta
$$

As we will see in the following, this variant of Tikhonov-Phillips algorithm insures the best possible order of accuracy in the power scale.

Denote by $\left\{u_{i}, v_{i}, \sigma_{i}\right\}$ the singular value decomposition for a compact operator $A$, where $u_{i}, v_{i} \in H$ are the singular vectors and $\sigma_{i}>0$ are the singular values.

Lemma 2 Assume that $A \in \mathcal{H}_{\gamma}^{r}$ and that $x^{\dagger}$ obeys (9). Then for $\nu \in(0,1 / 2]$

$$
\left\|x^{\dagger}-x_{\alpha, n}^{\delta}\right\|_{H} \leq \frac{\delta}{2 \sqrt{\alpha}}+\alpha^{\nu} c_{\nu, \alpha}(v)+\frac{c_{r, \gamma, \rho} n 2^{-2 n r}}{\alpha},
$$

where $c_{r, \gamma, \rho}=2 c_{r, \gamma} \gamma \rho$,

$$
c_{\nu, \alpha}^{2}(v)=\sum_{i \geq 0}\left\{\frac{\alpha^{1-\nu} \sigma_{i}^{2 \nu}}{\left(\sigma_{i}^{2}+\alpha\right)}\left(v, u_{i}\right)\right\}^{2} \leq\left\{(1-\nu)^{1-\nu} \nu^{\nu} \rho\right\}^{2} .
$$


Proof. We follow the proof of Lemma 2.5 in [12]. First of all we note that

$$
\begin{gathered}
\left\|x^{\dagger}-x_{\alpha, n}^{\delta}\right\|_{H} \leq\left\|x^{\dagger}-\left(\alpha I+A^{*} A\right)^{-1} A^{*} y\right\|_{H}+ \\
+\left\|\left(\alpha I+A_{n}^{*} A_{n}\right)^{-1} A_{n}^{*}\left(y-y_{\delta}\right)\right\|_{H}+ \\
+\left\|\left(\alpha I+A^{*} A\right)^{-1} A^{*} y-\left(\alpha I+A_{n}^{*} A_{n}\right)^{-1} A_{n}^{*} y\right\|_{H} .
\end{gathered}
$$

Equation (9) and inserting the singular value decomposition yields

$$
\begin{gathered}
\left\|x^{\dagger}-\left(\alpha I+A^{*} A\right)^{-1} A^{*} y\right\|_{H}= \\
=\left\|\left(\alpha I+A^{*} A\right)^{-1}\left(\alpha x^{\dagger}+A^{*} A x^{\dagger}-A^{*} y\right)\right\|_{H}= \\
=\alpha\left\|\left(\alpha I+A^{*} A\right)^{-1} x^{\dagger}\right\|_{H} \leq c_{\nu, \alpha}(v) \alpha^{\nu} .
\end{gathered}
$$

Moreover, from standard estimates using the singular value decomposition of compact operator $T$ one knows:

$$
\begin{gathered}
\left\|\left(\alpha I+T^{*} T\right)^{-1}\right\|_{H \rightarrow H} \leq \alpha^{-1}, \quad\left\|\left(\alpha I+T^{*} T\right)^{-1} T^{*}\right\|_{H \rightarrow H} \leq \frac{1}{2 \sqrt{\alpha}} \\
\left\|\left(\alpha I+T^{*} T\right)^{-1} T^{*} T\right\|_{H \rightarrow H} \leq 1,
\end{gathered}
$$

where $T=A, A^{*}$ or $T=A_{n}, A_{n}^{*}$. Then

$$
\left\|\left(\alpha I+A_{n}^{*} A_{n}\right)^{-1} A_{n}^{*}\left(y-y_{\delta}\right)\right\|_{H} \leq \frac{\delta}{2 \sqrt{\alpha}} .
$$

On the other hand, from (9), (26) and Lemma 1 we know that

$$
\begin{gathered}
\left\|\left(\alpha I+A^{*} A\right)^{-1} A^{*} y-\left(\alpha I+A_{n}^{*} A_{n}\right)^{-1} A_{n}^{*} y\right\|_{H} \leq \\
\leq\left\|\left(\alpha I+A_{n}^{*} A_{n}\right)^{-1}\left(A_{n}^{*} A_{n}-A^{*} A\right)\left(\alpha I+A^{*} A\right)^{-1} A^{*} y\right\|_{H}+ \\
+\left\|\left(\alpha I+A_{n}^{*} A_{n}\right)^{-1}\left(A^{*}-A_{n}^{*}\right) y\right\|_{H} \leq \\
\leq \alpha^{-1}\left\|A_{n}^{*} A_{n}-A^{*} A\right\|_{H \rightarrow H}\left\|\left(\alpha I+A^{*} A\right)^{-1} A^{*} A x^{\dagger}\right\|_{H}+ \\
+\alpha^{-1}\left\|\left(A^{*}-A_{n}^{*}\right) A x^{\dagger}\right\|_{H} \leq 2 \alpha^{-1} c_{r, \gamma} n 2^{-2 n r}\left\|x^{\dagger}\right\|_{H} \leq \\
\leq 2 c_{r, \gamma} \gamma^{2 \nu} \rho \alpha^{-1} n 2^{-2 n r} \leq c_{r, \gamma, \rho} \frac{n 2^{-2 n r}}{\alpha} .
\end{gathered}
$$


The assertion of the lemma follows from (24)-(27).

We now continue to analyse the convergence properties of the proposed adaptive scheme by following the standard lines of proof; i.e. we first show that the proposed stopping criterion with noisy data yields a regularization parameter $\alpha$ which would have been also obtained by a related discrepancy principle with perfect data.

Lemma 3 If $\alpha=\alpha_{N}$ and $n=n\left(\alpha_{N}, \delta\right)$ are chosen within the framework of algorithm (19)-(23) for $A \in \mathcal{H}_{\gamma}^{r}$ then there exist $d_{1}, d_{2}>0$ such that

$$
d_{1} \delta \leq\left\|A x_{\alpha}-y\right\|_{H} \leq d_{2} \delta
$$

where $x_{\alpha}=\left(\alpha I+A^{*} A\right)^{-1} A^{*} y$.

Proof. We follow the proof of Lemma 7 and Lemma 10 in [10].

We put $R_{\alpha}(T)=\left(\alpha I+T^{*} T\right)^{-1} T^{*}$ and $x_{\alpha}^{\delta, n}=R_{\alpha}\left(A_{n}\right) y^{\delta}$. Then

$$
\begin{gathered}
A x_{\alpha}-y=A R_{\alpha}(A) y-y= \\
\left(A R_{\alpha}(A)-A_{n} R_{\alpha}\left(A_{n}\right)\right) y+\left(I-A_{n} R_{\alpha}\left(A_{n}\right)\right)\left(y-y_{\delta}\right)+\left(A_{n} x_{\alpha, n}^{\delta}-y_{\delta}\right) .
\end{gathered}
$$

Keeping in mind that

$$
\begin{gathered}
T\left(\alpha I+T^{*} T\right)^{-1}=\left(\alpha I+T T^{*}\right)^{-1} T, \\
T^{*}\left(\alpha I+T T^{*}\right)^{-1}=\left(\alpha I+T^{*} T\right)^{-1} T^{*},
\end{gathered}
$$

we have

$$
\begin{gathered}
\left(A R_{\alpha}(A)-A_{n} R_{\alpha}\left(A_{n}\right)\right) y=\left(A A^{*}\left(\alpha I+A A^{*}\right)^{-1}-A_{n} A_{n}^{*}\left(\alpha I+A_{n} A_{n}^{*}\right)^{-1}\right) y \\
=\left(A A^{*}-A_{n} A_{n}^{*}\right)\left(\alpha I+A A^{*}\right)^{-1} y-A_{n} A_{n}^{*}\left(\alpha I+A_{n} A_{n}^{*}\right)^{-1}\left(A A^{*}-A_{n} A_{n}^{*}\right)\left(\alpha I+A A^{*}\right)^{-1} y \\
=\left(\alpha I+A_{n} A_{n}^{*}\right)^{-1}\left\{\left(\alpha I+A_{n} A_{n}^{*}\right)-A_{n} A_{n}^{*}\right\}\left(A A^{*}-A_{n} A_{n}^{*}\right)\left(\alpha I+A A^{*}\right)^{-1} y \\
=\alpha\left(\alpha I+A_{n} A_{n}^{*}\right)^{-1}\left(A A^{*}-A_{n} A_{n}^{*}\right)\left(\alpha I+A A^{*}\right)^{-1} y .
\end{gathered}
$$

Using this formula, (26) and Lemma 1, we obtain the following estimate for any $\alpha=\alpha_{m}$ and $n=n\left(\alpha_{m}, \delta\right)$

$$
\begin{gathered}
\left\|\left(A R_{\alpha}(A)-A_{n} R_{\alpha}\left(A_{n}\right)\right) y\right\|_{H}= \\
=\alpha\left\|\left(\alpha I+A_{n} A_{n}^{*}\right)^{-1}\left(A A^{*}-A_{n} A_{n}^{*}\right)\left(\alpha I+A A^{*}\right)^{-1} A x^{\dagger}\right\|_{H} \leq
\end{gathered}
$$




$$
\leq \frac{c_{r, \gamma} n 2^{-2 n r} \gamma^{2 \nu} \rho}{2 \sqrt{\alpha}} \leq \frac{c_{r, \gamma, \rho} n 2^{-2 n r}}{4 \sqrt{\alpha}}=\delta .
$$

Moreover, it is easy to see that

$$
\begin{gathered}
\left\|\left(I-A_{n} R_{\alpha}\left(A_{n}\right)\right)\left(y-y_{\delta}\right)\right\|_{H} \leq \|\left(I-\left(\alpha I+A_{n} A_{n}^{*}\right)^{-1} A_{n} A_{n}^{*}\left\|_{H \rightarrow H}\right\| y-y_{\delta} \|_{H} \leq\right. \\
\leq \alpha \delta\left\|\left(\alpha I+A_{n} A_{n}^{*}\right)^{-1}\right\|_{H \rightarrow H} \leq \delta .
\end{gathered}
$$

If $\alpha=\alpha_{N}$ satisfies (23) then combining (29)-(30) we have

$$
\left\|A x_{\alpha}-y\right\|_{H} \leq(d+2) \delta .
$$

On the other hand, the same steps as in the proof of Lemma 10 [10] lead to the inequality

$$
\left\|A x_{\alpha_{N}}-y\right\|_{H}=\left\|A x_{q \alpha_{N-1}}-y\right\|_{H} \geq q\left\|A x_{\alpha_{N-1}}-y\right\|_{H},
$$

where $q$ is the denominator of geometric progression $\left\{\alpha_{m}: \alpha_{m}=q^{m} \alpha_{0}, m=\right.$ $1,2, \ldots\}$, which contains $\alpha_{N}$. But for $\alpha=\alpha_{N-1}$ and $n=n\left(\alpha_{N-1}, \delta\right)$ from the definition of algorithm (19)-(23) it follows

$$
\left\|A_{n} x_{\alpha_{N-1}, n}^{\delta}-y_{\delta}\right\|_{H}>d \delta .
$$

Then combining similarly (29)-(30) for $\alpha=\alpha_{N-1}, \quad n=n\left(\alpha_{N-1}, \delta\right)$, by inverse triangle inequality we have

$$
\left\|A x_{\alpha_{N-1}}-y\right\|_{H} \geq\left\|A_{n} x_{\alpha_{N-1}, n}^{\delta}-y_{\delta}\right\|_{H}-2 \delta>(d-2) \delta .
$$

Now by virtue of (31), (32) we find

$$
\left\|A x_{\alpha_{N}}-y\right\|_{H}>q(d-2) \delta .
$$

Thus, we obtain the assertion of the lemma for $d_{1}=q(d-2), d_{2}=d+2$.

\section{Complexity of the adaptive discretization scheme}

Theorem 2 The optimal order of accuracy in the power scale $\delta^{2 \nu /(2 \nu+1)}$ on the classes $\Phi_{\gamma, \rho}^{r, \nu}, 0<\nu \leq 1 / 2$, is realized by the algorithm (19)-(23). 
Proof. From Lemma 2 it follows that for any $\alpha=\alpha_{m}$ and $n=n\left(\alpha_{m}, \delta\right)$

$$
\left\|x^{\dagger}-x_{\alpha, n}^{\delta}\right\|_{H} \leq \frac{\delta}{2 \sqrt{\alpha}}+\alpha^{\nu} c_{\nu, \alpha}(v)+\frac{4 \delta}{\sqrt{\alpha}}=\frac{9 \delta}{2 \sqrt{\alpha}}+\alpha^{\nu} c_{\nu, \alpha}(v) .
$$

Moreover, one should note that inserting the singular value decomposition shows (see,e.g., [13]) that

$$
\left\|A x_{\alpha}-y\right\|_{H}^{2}=\alpha^{2 \nu+1} d_{\alpha, \nu}^{2}(v),
$$

where $d_{\alpha, \nu}(v)$ itself is bounded for $0<\nu \leq 1 / 2$ and

$$
c_{\nu, \alpha}(v)\left[d_{\alpha, \nu}(v)\right]^{-2 \nu /(2 \nu+1)} \leq c .
$$

Now if $\alpha=\alpha_{N}$ satisfies (23) and $n$ is chosen according to (19) for $\alpha_{m}=\alpha_{N}$ then from Lemma 3 and (34), (35) one sees that

$$
\begin{gathered}
\frac{\delta}{\sqrt{\alpha}}=\delta\left(\frac{d_{\alpha, \nu}(v)}{\left\|A x_{\alpha}-y\right\|_{H}}\right)^{1 /(2 \nu+1)} \leq \delta\left(\frac{d_{\alpha, \nu}(v)}{d_{1} \delta}\right)^{1 /(2 \nu+1)} \leq c \delta^{2 \nu /(2 \nu+1)}, \\
\alpha^{\nu} c_{\nu, \alpha}(v)=c_{\nu, \alpha}(v)\left(\frac{\left\|A x_{\alpha}-y\right\|_{H}}{d_{\alpha, \nu}(v)}\right)^{2 \nu /(2 \nu+1)} \leq \\
\leq c_{\nu, \alpha}(v)\left[d_{\alpha, \nu}(v)\right]^{-2 \nu /(2 \nu+1)}\left(d_{2} \delta\right)^{2 \nu /(2 \nu+1)} \leq c \delta^{2 \nu /(2 \nu+1)}
\end{gathered}
$$

The assertion of the theorem follows from (33), (36), (37) .

Let us denote by $\operatorname{card}(E q)$ the number of linear algebraic equations in the system corresponding to (22). Using the representation

$$
\begin{gathered}
A_{n}=\sum_{j=1}^{n}\left(P_{j}-P_{j-1}\right) A P_{2 n-j}+\sum_{j=1}^{n} P_{2 n-j} A\left(P_{j}-P_{j-1}\right)+ \\
+P_{2 n} A P_{0}+P_{0} A P_{2 n}-P_{n} A P_{n}
\end{gathered}
$$

and (5) we get the estimate

$$
\begin{aligned}
& \operatorname{card}(E q)=\operatorname{rank} A_{n}^{*}=\operatorname{rank} A_{n} \leq \operatorname{rank}\left(\sum_{j=1}^{n}\left(P_{j}-P_{j-1}\right) A P_{2 n-j}\right)+ \\
& \quad+\operatorname{rank}\left(\sum_{j=1}^{n} P_{2 n-j} A\left(P_{j}-P_{j-1}\right)\right) \leq 2 \operatorname{rank} P_{n} \sim \operatorname{dim} V_{n} \sim 2^{s n} .
\end{aligned}
$$


Now we estimate the number card(IP) of inner products of the form (20), (21) required to construct an approximate solution $x_{\alpha_{m}, n}^{\delta}$. From (5), (20), (21) it follows

$$
\begin{gathered}
\operatorname{card}(I P) \leq \sum_{j=0}^{2 n} \operatorname{dim} V_{j} \cdot \operatorname{dim} V_{2 n-j}+\operatorname{dim} V_{2 n} \sim \\
\sim \sum_{j=0}^{2 n} 2^{s j} 2^{(2 n-j) s}+2^{2 n s} \sim n 2^{2 n s} .
\end{gathered}
$$

To illustrate the advantages of proposed adaptive discretization scheme we assume that $\alpha_{N}$ satisfying (23) has the order $\delta^{2-2 \lambda}$ for some $\lambda \in(0,1 / 2)$. It is sufficiently natural assumption because (see, e.g. [6]) the regularization parameter $\alpha$ is normally chosen in dependence of $\delta$ such that

$$
\lim _{\delta \rightarrow 0} \frac{\delta^{2}}{\alpha}=0, \quad \lim _{\delta \rightarrow 0} \alpha=0 .
$$

Keeping in mind (19) for $\alpha_{m}=\alpha_{N} \sim \delta^{2-2 \lambda}$ and

$$
n 2^{-2 n r} \sim \delta^{2-\lambda}
$$

we obtain the following estimates

$$
\begin{aligned}
& \operatorname{card}(E q) \leq c 2^{n s} \sim \delta^{-(2-\lambda) s /(2 r)} \log ^{s /(2 r)} \frac{1}{\delta}, \\
& \operatorname{card}(I P) \leq c n 2^{2 n s} \sim \delta^{-(2-\lambda) s / r} \log ^{1+s / r} \frac{1}{\delta} .
\end{aligned}
$$

Then the total number of inner products of the form (13) required to achieve the optimal order of accuracy in the power scale within the framework of algorithm (19)-(23) is no more than

$$
\operatorname{card}(I P) \cdot N \leq c \log \frac{1}{\delta} n 2^{2 n s} \sim \delta^{-(2-\lambda) s / r} \log ^{2+s / r} \frac{1}{\delta} .
$$

Moreover, from (36) it follows that if $\alpha_{N}$ satisfies (23) then $\left(\delta \sqrt{\alpha_{N}}\right)^{-1} \leq$ $c \delta^{-2(\nu+1) /(2 \nu+1)}$. Therefore

$$
\operatorname{Card}(I P) \cdot N \leq c\left(\delta \sqrt{\alpha_{N}}\right)^{-s / r} \log ^{2+s / r}\left(\delta \sqrt{\alpha_{N}}\right)^{-1} \leq c \delta^{-\frac{2 s(\nu+1)}{r(2 \nu+1)}} \log ^{2+s / r} \frac{1}{\delta} .
$$

When these relations are compared with (14) it is apparent that for the classes $\Phi_{\gamma, \rho}^{r, \nu}, 0<\nu \leq 1 / 2$, the adaptive discretization scheme (19)-(23) is more efficient than traditional non-adaptive approach to discretization (10)(12). 


\section{Numerical example}

A standard example for inverse ill-posed problems is given by (see [9])

$$
A x=y
$$

with the compact operator

$$
A: L^{2}([0,1]) \longrightarrow L^{2}([0,1]), \quad x \longmapsto \int_{0}^{1} k(s, t) x(t) d t,
$$

where

$$
k(s, t)=\left\{\begin{array}{ll}
t(1-s) & \text { for } t \leq s \\
s(1-t) & \text { for } s \leq t
\end{array} .\right.
$$

As the solution $x$ we take $x(s)=s(1-s)$ which yields

$$
y(s)=A x(s)=\frac{1}{12}\left(s^{4}-2 s^{3}+s\right) .
$$

This operator lies in the considered class $A \in \mathcal{H}_{\gamma}^{r}$ with $r=2$. We now compare the results of the full discretized operator $A_{n}=P_{n} A P_{n}$ with those obtained with our algorithm (19)-(23). The discrepancy principle was used in both cases with $d=2$ and the sequence of regularization parameters $\alpha_{m}=q^{m} \alpha_{0}$ was obtained with $\alpha_{0}=1$ and $q=0.8$. Moreover the constant $4 c_{r, \gamma, \rho}^{-1}$ in equation (19) was set to 1 for simplicity.

The discretization spaces $V_{j}$ were obtained as in Chapter 2 with the Haarwavelet basis, i.e. $s=2$ gives the desired level of approximation. The proposed adaptive scheme shows its advantage for small noise levels, i.e. for comparatively large dimensions $n$. Figure 2 displays the reconstructions with both methods for a noise level of $0,5 \%$, the reconstruction error $\left\|x^{\dagger}-x_{\alpha, n}^{\delta}\right\|$ was 0.0145 for the adaptive scheme and 0.0165 for the full discretization. However the number of scalar products required to construct the discretized matrix $A_{n}$ was 764 compared to 16384 . Table 1 displays the results for different noise levels, the value of "dimension" refers to finest level of discretization, which was used for the final value of $\alpha$. 


\begin{tabular}{|l|l|r|r|r|}
\hline noise level & method & $\alpha$ & dimension & number of scalar products \\
\hline \hline $5 \%$ & adaptive & 1 & $2^{4}$ & 36 \\
& full & 1 & $2^{2}$ & 16 \\
\hline $1 \%$ & adaptive & 0.0024 & $2^{6}$ & 164 \\
& full & 0.0059 & $2^{6}$ & 4096 \\
\hline $0.5 \%$ & adaptive & 0.0010 & $2^{8}$ & 764 \\
& full & 0.0038 & $2^{7}$ & 16384 \\
\hline
\end{tabular}

Table 1: The adaptive discretization scheme shows its advantage for small noise levels, the computation of $A_{n}$ requires substantially less scalar products, despite the smaller value of the $\alpha$, which was determined by Morozov's discrepancy principle.

Figure 1: Left: result obtained with the adaptive strategy and noise level $0.5 \%$, right: result obtained with full discretization and noise level $0.5 \%$
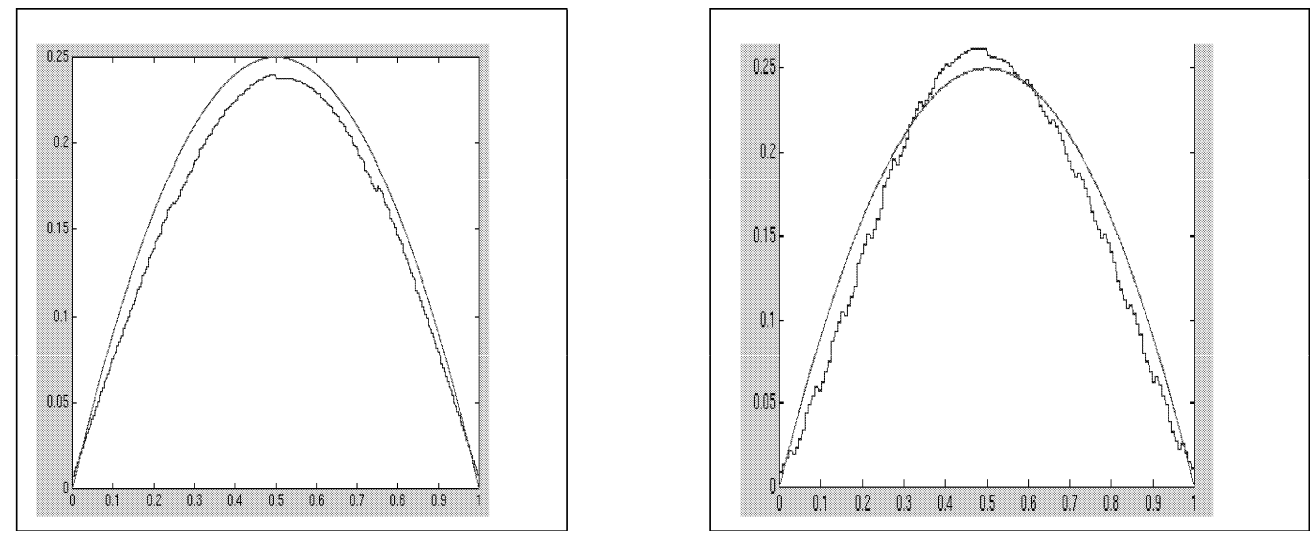

\section{References}

[1] Bakushinskii, A.B. (1965), On some numerical method for solving integral equations of the first kind, USSR Comput.Maths.Math.Phys. 5, 744-749.

[2] G. Beylkin, R. Coifman, V. Rokhlin, (1991) Fast Wavelet Transform and Numerical Algorithms I, Comm. Pure Appl. Math., 44, pp. 141-183. 
[3] Dahmen, W., Kunoth, A.,and Schneider, R. (1996), Operator equations, multiscale concepts and complexity, In Renegar, J., Shub, M., Smale, S. (eds) Lectures in Applied Mathematics, 32, Amer.Math.Soc. 225-261.

[4] Dicken, D., Maass, P. (1996), Wavelet-Galerkin Methods for Ill-posed Problems, J. Inv. and Ill-posed Probl., Vol. 4, No.3., pp. 203-222.

[5] Donoho, D. L. (1992), Nonlinear solution of linear inverse problems by wavelet-vaguelette decomposition Technical Report, Stanfrod University, Department of Statistics.

[6] Engl, H. W. (1981), Necessary and sufficient conditions for convergence of regularization methods for solving linear operator equations of the first kind, Numer.Funct.Anal.Optim. 3, 201-222.

[7] Frommer, A., Maass, P. (1998), Fast CG-based methods for Tikhonov regularization, accepted for publication SIAM J. Sci. Comp..

[8] Ivanov, V.K., Vasin, V.V., Tanana, V.P. (1978) "Theory of linear illposed problems and its applications," Nauka, Moscow. [Russian]

[9] Louis, A. K., 1992, Inverse und schlecht gestellte Probleme, Teubner, Stuttgart.

[10] Maaß, P.,and Rieder, A. (1997), Wavelet-Accelerated Tikhonov-Phillips regularization with applications, submitted for publication, Preprint University of Potsdam.

[11] Morozov, V. A. (1966), On the solution of functional equations by the method of regularization, Soviet Math.Doklady 7, 414-417.

[12] Neubauer, A. (1988), An a posteriori parameter choice for Tikhonov regularization in the presence of modeling error, Apl.Num.Math. 4, 507519.

[13] Neubauer, A. (1988), An a posteriori parameter selection choice for Tikhonov regularization in Hilbert scales leading to optimal convergence rates, SIAM J.Numer.Anal. 25, 1313-1326.

[14] Pereverzev, S.V., Optimization of Projection Methods for Solving IllPosed Problems , Computing 55 (1995) 
[15] Pereverzev, S.V. (1996), Optimization of Methods for Approximate Solution of Operator Equations, Nova Science Publishers, New York.

[16] Plato, R., and Vainikko, G. (1990), On the Regularization of Projection Methods for solving Ill-posed Problems, Numer.Math. 57, 63-79.

[17] Schock, E. (1984), On the asymptotic order of accuracy of Tikhonov regularization, J.Optim. Theory Appl. 44, 95-104.

[18] Vainikko, G.M. (1982), The discrepancy principle for a class of regularization methods, USSR Comput.Maths.Math.Phys. 22, 1-19. 